\title{
Recent heavy flavor measurements from PHENIX at RHIC
}

\author{
Takashi Hachiya ${ }^{1, a}$ for the PHENIX collaboration \\ ${ }^{1}$ RIKEN BNL Research Center
}

\begin{abstract}
Heavy flavor is a useful probe to study the property of the strongly coupled quark gluon plasma created in high-energy heavy-ion collisions. They are produced in the early stage of the collisions, and then propagate though QGP. Therefore, they carry information of QGP. PHENIX measures the separated bottom and charm production using the precise tracking by the silicon vertex detector installed at mid-rapidity and forward rapidity. In this article, we present the recent results of separated charm and bottom modification in $\mathrm{Au}+\mathrm{Au}$ and $\mathrm{Cu}+\mathrm{Au}$ collisions, and charmonia production in $p+\mathrm{Al}$ and $p+\mathrm{Au}$ collisions.
\end{abstract}

\section{Introduction}

Hadrons carrying heavy quarks, bottoms and charms are crucial probes to study the nature of quark gluon plasma (QGP) created in high energy heavy ion collisions. Because of their large masses $\left(M_{b} \approx\right.$ $4.2 \mathrm{GeV} / c^{2}$ and $\left.M_{c} \approx 1.3 \mathrm{GeV} / c^{2}\right)$, heavy quarks are mainly produced via parton-parton collisions at the initial stage of the reaction. Once produced, heavy quarks then interact with QGP when they traverse it. Then, they suffer the QGP effects such as energy loss, flow effect and dissociation of their pairs. Therefore, the modification of the heavy quark production is sensitive to the property of QGP. Heavy quarks are also modified by cold nuclear matter (CNM) effects. To disentangle QGP effects from CNM effects, it is necessary to study the heavy flavor production in $p+p, p+\mathrm{A}$ and $\mathrm{A}+\mathrm{A}$ collisions.

PHENIX studied a variety of open and closed heavy favor productions in $p+p$ and $\mathrm{Au}+\mathrm{Au}$ collisions. We previously measured a strong suppression and a substantial flow of heavy flavors in the measurement of single electrons from semi-leptonic decays of bottom and charm hadrons[1, 2]. The results are not well described by the expectation of energy loss via gluon radiation such as the dead cone effect[3]. PHENIX $\psi(2 S)$ measurement in central $d+$ Au collisions at mid-rapidity showed stronger suppression than the $J / \psi$ but similar suppression in peripheral collisions[4]. The sequential suppression of $\psi(2 S)$ and $J / \psi$ can not be explained by the nuclear absorption.

A Barrel and Forward Silicon Vertex Tracker (VTX and FVTX) were installed in PHENIX in 2011 and 2012. VTX (FVTX) covers $|y|<1.2(1.2<|y|<2.2)$ in rapidity and almost $2 \pi$ (full) in azimuth. These silicon detectors provide precise displaced tracking and better momentum resolution. The precise tracking enables to measure the bottom and charm components separately in the single electron measurement by using the difference of their decay lengths. Better mass resolution allows to separate $J / \psi$ and $\psi(2 S)$ in the measurement of lepton pairs from their decays. In this article, we

\footnotetext{
a e-mail: hachiya@rcf.rhic.bnl.gov
} 

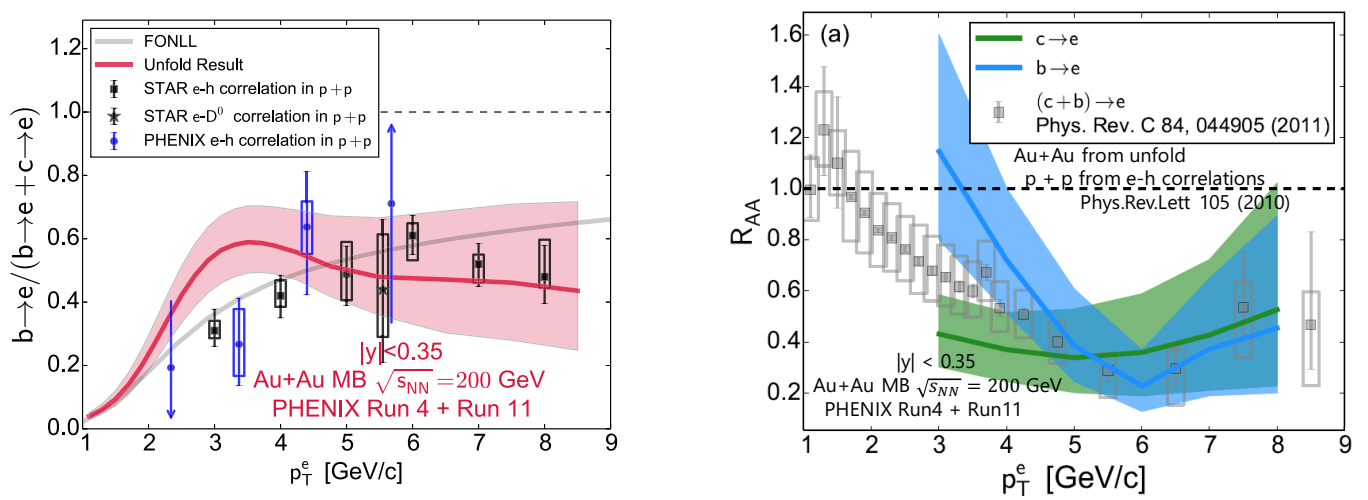

Figure 1. (Left) The bottom electron fraction in minimum bias $\mathrm{Au}+\mathrm{Au}$ collisions at $\sqrt{s_{N N}}=200 \mathrm{GeV}[5]$, which are compared with the FONLL calculation[8]. Also the $p+p$ result at same energy from PHENIX[6] and STAR[7] are shown. (Right) $R_{A A}$ for electrons from separated bottom and charm hadron decays as well as inclusive heavy flavor electrons.

report on separated bottom and charm yields in mid and forward rapidity in heavy ion collisions and $J / \psi$ and $\psi(2 S)$ production in $p+$ A collisions.

\section{Separated bottom and charm yields in Au + Au collisions}

We utilize the difference of the decay lengths of $B$ and $D$ mesons $\left(c \tau_{B^{0}} \approx 455 \mu \mathrm{m}\right.$ and $\left.c \tau_{D^{0}} \approx 123 \mu \mathrm{m}\right)$ in order to separate bottom and charm components in the single electron measurement. Electrons are identified using a ring-imaging Čerenkov detector and the energy-momentum matching. VTX measures the distance of closest approach $(D C A)$ of charged tracks from the collision vertex. The DCA distribution of inclusive electrons contains a large amount of backgrounds that originate from non-desired physics processes such as Dalitz decays of $\pi^{0}$ and $\eta$, photon conversions as well as detector effects such as mis-identified hadrons and high multiplicity background. Those backgrounds are estimated and subtracted using the event driven method and the PHENIX detector simulations. The heavy flavor signals in the DCA distribution are extracted, and the bottom and charm components are separated by the unfolding method based on Bayesian inference technique. The analysis procedure is described in detail[5].

Figure. 1 (left) shows a fraction of electrons from bottom decays to inclusive heavy flavors. The red curve and pink band represent the central value and systematic uncertainties. The bottom electron fraction is compared with the $p+p$ results [6, 7] and FONLL calculation[8]. It is found that the $\mathrm{Au}+$ Au result shows a apparent peak at $p_{T} \sim 3 \mathrm{GeV} / c$ but $p+p$ results increase smoothly with $p_{T}$ and is consistent with the FONLL within uncertainties.

The nuclear modification factor $\left(R_{A A}\right)$ for electrons from bottom and charm hadron decays are calculated using the bottom electron fraction in $\mathrm{Au}+\mathrm{Au}$ and $p+p$, and $R_{A A}$ of inclusive heavy flavor electrons[2], as shown in the right panel of Fig. 1. We find that electrons from bottom decays are less suppressed than that from charm decays for $p_{T}<4 \mathrm{GeV} / c$ and similarly suppressed for higher $p_{T}$. This result is qualitatively consistent with the expectations from the mass dependence of energy $\operatorname{loss}[3,9]$. 

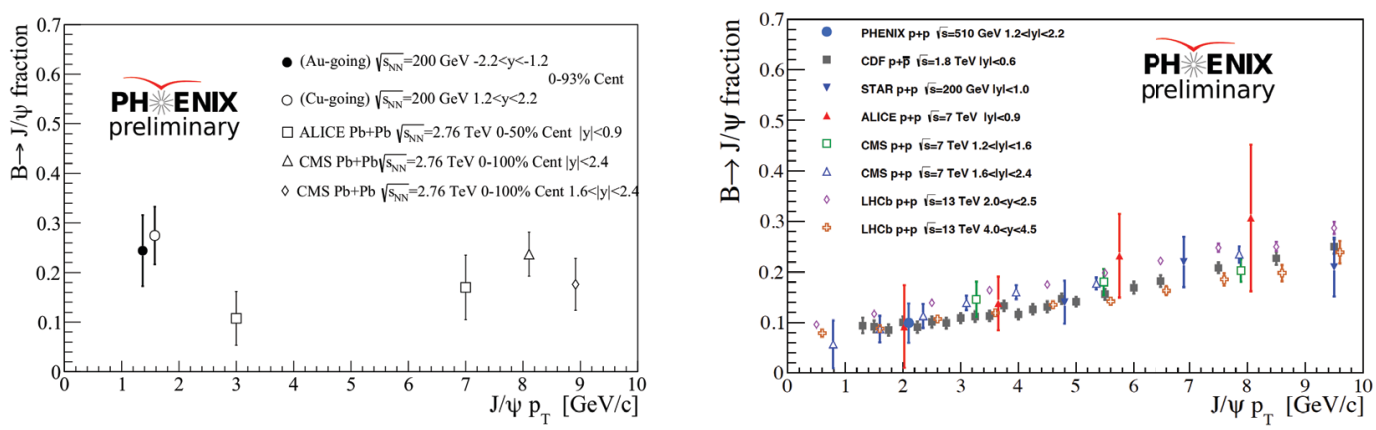

Figure 2. The fraction of $B \rightarrow J / \psi$ relative to inclusive $J / \psi$ in minimum bias $\mathrm{Cu}+\mathrm{Au}$ collisions at $\sqrt{s_{N N}}=$ $200 \mathrm{GeV}$ (left) and $p+p$ collisions at $\sqrt{s_{N N}}=510 \mathrm{GeV}$ (right) The $\mathrm{Cu}+\mathrm{Au}$ result are compared with the LHC results.

\section{Open bottom measurement from $B \rightarrow J / \psi$ decays in $\mathrm{Cu}+\mathrm{Au}$ collisions}

$J / \psi$ from $B$ meson decays is a direct measurement of open bottom production. PHENIX measure the signal via $B \rightarrow J / \psi \rightarrow \mu \mu$ at forward and backward rapidity in $200 \mathrm{GeV} \mathrm{Cu}+\mathrm{Au}$ collisions. $J / \psi$ is reconstructed from unlike sign di-muon pairs. DCA of single muons from $J / \psi$ decays is measured by FVTX. The DCA distribution is asymmetric for the B meson decays because the negative side of the DCA has better detector acceptance, while prompt $J / \psi$ from the collision vertex is symmetric. The difference of their shapes in the DCA distribution is used to identify muons from the $B \rightarrow J / \psi$ decays. The DCA distribution is fit with the template shapes of $B \rightarrow J / \psi$ and prompt $J / \psi$ components to extract the $B$ decay signal. Their template shapes are determined by PYTHIA + PHENIX detector simulation.

Figure 2 shows the preliminary result of the fraction of $B \rightarrow J / \psi$ decays relative to inclusive $J / \psi$ in $200 \mathrm{GeV} \mathrm{Cu}+\mathrm{Au}$ (left) and $510 \mathrm{GeV} p+p$ (right) collisions. In the left panel, the fraction is consistent for $\mathrm{Cu}$ going and $\mathrm{Au}$ going direction but larger than the CMS and ALICE results[10, 11]. The difference is attributed to a smaller suppression of prompt $J / \psi$ at the LHC energy. On the other hand, the $p+p$ result in the right panel follows the same trend with the world data. The nuclear modification factor $R_{C u A u}$ of $B \rightarrow J / \psi$ is calculated using these fractions and the $R_{C u A u}$ of inclusive $J / \psi$. Here, we assume that the fraction in $200 \mathrm{GeV} p+p$ collisions is 0.1 based on the world average data. Figure 3 shows $R_{C и A u}$ of $B \rightarrow J / \psi$. The $R_{C u A u}$ of $B \rightarrow J / \psi$ are consistent with unity within uncertainties for both $\mathrm{Cu}$ and $\mathrm{Au}$ going direction. The result indicates that the bottom suffers smaller suppression at low $p_{T}$ at RHIC energy. In the comparison, it is found that the $R_{C u A u}$ follows the same trend with the LHC results, where the suppression is less at low $p_{T}$ and more at high $p_{T}$.

\section{Closed heavy flavor in $\mathbf{p}+\mathbf{A}$ collisions : $J / \psi$ and $\psi(2 S)$}

The $J / \psi$ and $\psi(2 S)$ productions in $p+$ A collisions are a good tool to study the CNM effect. In the CNM effect, the initial state effects such as gluon shadowing and Cronin effect should be similar for both $J / \psi$ and $\psi(2 S)$, while the final state effects such as nuclear breakup effect are different because of their binding energies. PHENIX studies the $J / \psi$ and $\psi(2 \mathrm{~S})$ production in $200 \mathrm{GeV} p+p, p+\mathrm{Al}$ and $p+\mathrm{Au}$ collisions at forward and backward rapidity.

The double ratio of $\psi(2 \mathrm{~S})$ to $J / \psi$ yield in $p+\mathrm{A}$ collisions relative to the same ratio in $\mathrm{p}+\mathrm{p}$ collisions is used to quantify the modification of the production. Figure 4 shows the preliminary result of the 


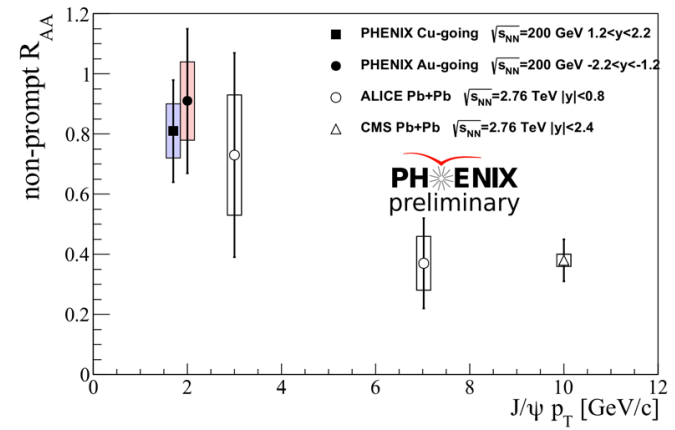

Figure 3. $R_{C u A u}$ for $B \rightarrow J / \psi$ is compared with the LHC results

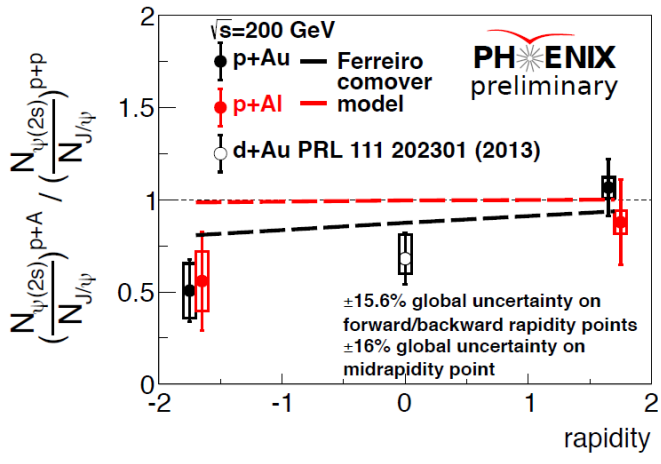

Figure 4. The double ratio of $\psi(2 \mathrm{~S})$ to $J / \psi$ in $200 \mathrm{GeV} p+\mathrm{Al}$ and $p+\mathrm{Au}$ collisions. The model calculation qualitatively describe the data.

double ratio in $p+\mathrm{Al}$ and $p+\mathrm{Au}$ collisions. The previous result at mid-rapidity in $d+\mathrm{Au}$ collisions is also plotted for the comparison. We find that the double ratio at forward rapidity, positive side, is consistent with unity within uncertainty. This indicates that the nuclear modifications at forward rapidity are comparable for $J / \psi$ and $\psi(2 S)$. However, the double ratio at backward rapidity, negative side, shows $\psi(2 S)$ is more suppressed than $J / \psi$ with a factor of 2 . Including the result at mid rapidity in $d+$ Au collisions, the rapidity dependence of the double ratios is clearly seen. We compared with the model including the interaction with the co-moving hadrons[12], and find that the model qualitatively describes the result.

\section{Summary}

PHENIX measured open and closed heavy flavor productions in $\mathrm{Au}+\mathrm{Au}, \mathrm{Cu}+\mathrm{Au}, p+\mathrm{A}$ collisions. VTX and FVTX enables us to separate the bottom and charm contributions in the single electron measurement in $\mathrm{Au}+\mathrm{Au}$ collisions and to identify $\mathrm{J} / \psi$ from $B$ meson decays in $\mathrm{Cu}+\mathrm{Au}$ collisions. The better mass resolution by FVTX provides the separation of $J / \psi$ and $\psi(2 S)$ in $p+A$ collisions. For the first time, we found the less suppression of electrons from bottom decays than that from charm decays for $3<p_{T}<4 \mathrm{GeV} / \mathrm{c}$ and similar suppression for higher $p_{T}$. The first result of $B \rightarrow J / \psi$ decays also shows the small suppression at low $p_{T}$ in $\mathrm{Cu}+\mathrm{Au}$ collisions. The sequential suppression of $\psi(2 \mathrm{~S})$ and $J / \psi$ is found at backward rapidity, which is described by the comoving hadron model. These results from the heavy flavor measurement shed a light on the QGP property and the CNM effect from different dimensions. We collected large amount of $\mathrm{Au}+\mathrm{Au}$ and $p+p$ data in 2014 to 2016. These new dataset should provide definitive measurements of open and closed heavy flavor productions.

\section{References}

[1] A. Adare et al. Energy Loss and Flow of Heavy Quarks in Au + Au Collisions at $\sqrt{s_{N N}}=200$ GeV Phys. Rev. Lett. 98172301 (2007)

[2] A. Adare et al. Heavy-quark production in $p+p$ and energy loss and flow of heavy quarks in $\mathrm{Au}$ + Au collisions at $\sqrt{s_{N N}}=200 \mathrm{GeV}$ Phys. Rev. C 84, 044905 (2011) 
[3] Y. L. Dokshitzer and D. E. Kharzeev Phys. Lett. B 519, 199 (2001)

[4] A. Adare et al. Nuclear Modification of $\Psi^{\prime}, \chi_{c}$, and $J / \Psi$ Production in $d+$ Au Collisions at $\sqrt{s_{N N}}$ = 200GeV Phys. Rev. Lett. 111, 202301 (2013)

[5] A. Adare et al. Single electron yields from semileptonic charm and bottom hadron decays in $\mathrm{Au}$ + Au collisions at $\sqrt{s_{N N}}=200 \mathrm{GeV}$ Phys. Rev. C 93034904 (2016)

[6] A. Adare et al. Measurement of Bottom versus Charm as a Function of Transverse Momentum with Electron-Hadron Correlations in $p+p$ Collisions at $\sqrt{s}=200 \mathrm{GeV}$ Phys. Rev. Lett. 103 082002 (2009)

[7] M. Aggarwal et al. Measurement of the Bottom contribution to non-photonic electron production in $p+p$ collisions at $\sqrt{s}=200 \mathrm{GeV}$ Phys. Rev. Lett. 105202301 (2010)

[8] M. Cacciari, P. Nason, and R.Vogt, QCD Predictions for Charm and Bottom Quark Production at RHIC Phys. Rev. Lett. 95122001 (2005)

[9] A. Adil and I. Vitev Collisional dissociation of heavy mesons in dense QCD matter Phys. Lett. B 649 139-146 (2007)

[10] S. Chatrchyan et al. Suppression of non-prompt $J / \psi$, prompt $J / \psi$, and $\Upsilon(1 \mathrm{~S})$ in $\mathrm{PbPb}$ collisions at $\sqrt{s_{N N}}=2.76 \mathrm{TeV}$, JHEP 05, 063 (2012)

[11] J. Adam et al. Inclusive, prompt and non-prompt $J / \psi$ production at mid-rapidity in $\mathrm{Pb}-\mathrm{Pb}$ collisions at $\sqrt{s_{N N}}=2.76 \mathrm{TeV}$, JHEP 07, 051 (2015)

[12] E. G. Ferreiro, Excited charmonium suppression in proton-nucleus collisions as a consequence of comovers, Phys. Lett. B 749 98-103 (2015) 